\title{
CIRCUMVENTION OF EXTRARETINAL PHOTORESPONSES IN ASSESSING RECOVERY OF VISION FOLLOWING OPTIC NERVE CRUSH IN GOLDFISH
}

\author{
ROGER E. DAVIS and BARBARA E. SCHLUMPF
}

Mental Health Research Institute, University of Michigan, Ann Arbor, MI 48109 (U.S.A.)

(Received February 10th, 1982)

(Revised version received June 7th, 1982)

(Accepted July 5th, 1982)

Key words: vision - extraretinal photoresponse - classical conditioning - goldfish, Carassius auratus - respiratory conditioning

SUMMARY

Earlier experiments indicated that a conditioned light stimulus that was used to investigate the recovery of vision following optic nerve crush could evoke an extraretinal photoresponse. The present experiments sought to identify a visual stimulus that does not evoke a response following removal of both eyes for use in experiments on optic nerve regeneration. A stimulus consisting of a slight up-down movement of a small ring of light, that was kept stationary between conditioning trials, was classically conditioned to shock in eyed fish and the conditioned response consisted of a suppression of branchial ventilation movements. Following bilateral enucleation or a sham operation the fish received additional sessions of conditioning trials over a period of 3 weeks. Postoperative responding to the moving light stimulus was blocked in the enucleated but not the sham-operated fish. When the ring of light was turned on and off as a conditioned stimulus, responding was extensively but not completely eliminated following enucleation. The investigation confirms that extraretinal photostimulation can be classically conditioned to shock in at least some goldfish, and it shows that such conditioning can be circumvented by using a small moving light as the conditioned stimulus. 


\section{INTRODUCTION}

Regeneration of optic nerve fibers in the goldfish is a potentially advantageous preparation for investigations of the effects of chemical substances on neuronal development. Regeneration can be induced by crushing the optic-nerve, and the rate of regeneration can be assessed by measuring the time to recovery of vision. In recent experiments, we measured the time to regeneration using as an index the recovery of a branchial ventilation response to a conditioned light stimulus (CS) [2]. Disturbingly, however, a control experiment with bilaterally enucleated fish revealed that the CS used to test for retinal input could evoke an extraretinal photoresponse indicating a possible source of error in measuring the rate of regeneration of a crushed optic nerve.

The regeneration experiments were done in unilaterally enucleated fish. The one-eyed fish were administered classical conditioning trials in which a $15 \mathrm{sec}$ presentation of a white light CS in darkness ended with the delivery of an intense electrical shock which served as the unconditioned stimulus (US). The response that was measured consisted of a suppression of branchial movements during the first $5 \mathrm{sec}$ of the light stimulus interval. Following conditioning the intact optic nerve was crushed to induce regeneration. The time of recovery of the suppression response (SR) to the light CS was measured by administering a brief session of conditioning trials every several days through postoperative Day 24 . The results showed that restoration of optic nerve connections sufficient to mediate the SR occurred within 17 days.

Extraretinal sensitivity to the light CS was detected in fish in which the intact optic nerve was cut, instead of crushed, and the eye was removed. These bilateral enucleated fish showed no SRs in the early postoperative sessions but some showed a delayed, or long-latency SR in trials on Day 17 through 24. The latency was $4-5 \mathrm{sec}$ or longer. The delayed, or so-called 'slow' SR did not occur when the stimulus light was blocked with opaque tape. Thus the slow SR was inferred to be evoked by photic rather than non-photic stimuli, such as electrical changes, that might accompany the operation of the lamp. Although the data suggested that retinal and extraretinal photoresponses may be distinguished based on whether the response is 'fast' or 'slow', the extraretinal variable should be excluded from investigations of optic nerve regeneration.

The objective of the present experiments was to identify a light CS that is strictly visual, that is one which can be conditioned to shock in eyed fish but not enucleated fish, and to confirm that extraretinal photoresponses are a possible source of error in optic nerve regeneration experiments. We assumed, based on previous investigations of behavioral and electrophysiological photoresponses in enucleated fish $[6,8]$, that behavioral sensitivity to light is greatly decreased in enucleated goldfish, and that the enucleate perceives light only diffusely. This suggested that minimizing the changes in gross luminance associated with the CS, 
and increasing the level of ambient light to decrease the sensitivity of the extraretinal photoreceptors, should decrease the probability of an extraretinal photoresponse. To minimize luminance cues we used linear light emitting diodes (LEDs) to produce a 'moving' light CS.

In the first of the two experiments that are to be described, fish selected for responding to the moving LED-CS in light were subsequently administered bilateral enucleation or a sham (control) operation followed by a sequence of postoperative sessions of conditioning trials. The sequence was similar to that used in the earlier regeneration experiments but the number of trials per session was increased to facilitate possible conditioning of the CS in the enucleates. The sessions were administered with or without the overhead illumination to investigate whether darkness results in an increased probability of response in enucleated fish. In the second experiment the response to a change-of-intensity CS was investigated in fish that were conditioned in darkness both pre- and postoperatively.

EXPERIMENT 1. RESPONSE TO A MOVING LED STIMULUS PRESENTED IN LIGHT OR DARKNESS

\section{Method}

\section{Fish}

Goldfish (Carassius auratus L.; family, Cyprinidae), 8-15 g, obtained from Ozark Fisheries, Stoutland, MO, were kept in groups in 38-1 tanks. Water temperature ranged from 24 to $26^{\circ} \mathrm{C}$. At the start of an experiment fish were transferred to individual 4-l home tanks partially submerged in a water bath which was heated to $30^{\circ} \mathrm{C}$. The water bath enclosure was illuminated $12 \mathrm{~h}$ daily (08.00-20.00 h) by a 33-W fluorescent Gro-Lamp (General Electric) located $1 \mathrm{~m}$ above the water surface. The home tank was flushed with fresh tap water several times a day, the water previously being dechlorinated, heated to $30^{\circ} \mathrm{C}$ and acidified with $\mathrm{HCl}$ to $\mathrm{pH} 7.0-7.5$. The fish were fed Purina trout chow or Tetra flake flood once or twice daily. The experiments were conducted during the months of May through September.

\section{Conditioning tank}

Fish were conditioned individually in 3 clear Plexiglas tanks $10 \mathrm{~cm}$ high, $10 \mathrm{~cm}$ wide and $45 \mathrm{~cm}$ long which were kept in a separate room. The tanks were filled to $7 \mathrm{~cm}$ with fresh water at the start of each session of trials. A removable fish holder [1] made of opaque, black Plexiglas, was used to restrain the fish in the center of the tank. Openings in the side of the fish compartment of the holder were covered with a membrane of clear polyethylene which permitted presentation of the light stimulus to either eye, and delivery of the electric shock (Fig. 1). In addition to four $40-\mathrm{W}$ fluorescent lamps located on the ceiling of the 


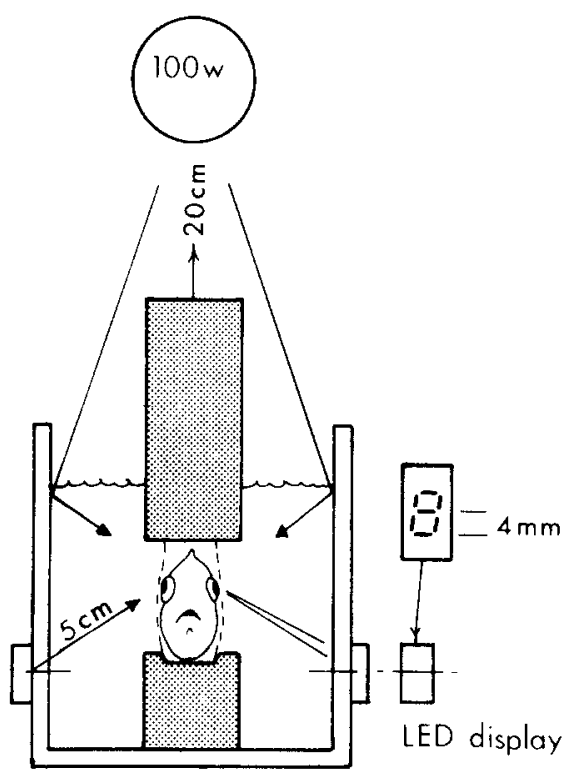

Fig. 1. The experimental tank showing the location of the two LED displays and of the overhead adapting light. The shock electrodes are omitted.

room, the tank was lighted by a $100-\mathrm{W}$ incandescent lamp centered above the fish holder. Branchial ventilation movements were detected by the thermistor method [7]. The thermistor was placed near the fish's mouth [1], and its output was registered with an ink-writing polygraph, which was kept in an adjoining room.

\section{Moving LED-CS}

A $4 \times 4 \mathrm{~mm}$ rectangular ring of red light produced by illuminating one loop of a figure ' 8 ' display of light emitting diodes (LED; Dial Light No. 7450017) was used. The display was mounted on the outside surface of the tank wall (Fig. 1), and it was visible to the fish through an opening in a dull white-colored plastic screen which was placed on the inside surface of the wall. The screen was illuminated by the overhead lights and it filled most of the fish's field of view. The intensity of the light which was reflected from the screen toward the fish holder was $40 \mathrm{ft}$-lamberts as measured with an S.E.I. photometer. The moving stimulus was produced by alternately illuminating the upper or lower loop of the ' 8 '. To evaluate the brightness of the LED ring, the display was placed $5 \mathrm{~cm}$ from the screen. The intensity of the reflected light was $1.5 \mathrm{ft}$-lamberts. The lower ring was kept lit during the intertrial interval. The duration of the CS interval was $15 \mathrm{sec}$. During this interval the light was alternated from the lower to the upper ring every $250 \mathrm{msec}$ (Fig. 2) by a microprocessor which controlled a double-throw-relay. Presentation of the stimulus to experimentally naive goldfish did not evoke changes in branchial movements. 


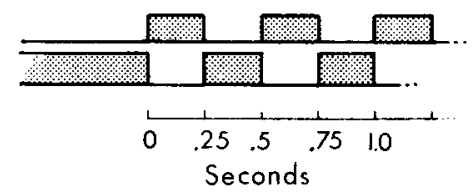

\begin{tabular}{ll}
\hline LEDCS & $15 \mathrm{~s}$ \\
\hline US & $5 \mathrm{~s}$ \\
\hline
\end{tabular}

Fig. 2. The operation of the LED display in producing the moving stimulus is diagrammed at the top of the figure; the lower trace represents the lower ring of the figure ' 8 ' display. The ring was kept on during the intertrial interval and turned off and on in alternation with the upper ring during the trial. The schedule of experimental stimuli is shown at the bottom of the figure.

\section{Mechanical conditioned stimulus (MCS)}

A mechanical stimulus consisting of a 1/sec tapping noise was presented during the last $5 \mathrm{sec}$ of the moving LED-CS interval (Fig. 2). A rotary solenoid attached to the end of the tank was activated for $200 \mathrm{msec}$ to produce the tap. A MCS was similarly used in the initial study [2] to maintain conditioning during the postoperative phase of a regeneration experiment wherein the fish is deprived of retinal input. While the advantage of using a non-photic CS remains to be demonstrated, the procedure was retained for experimental consistency. Presentation of the MCS alone elicited a momentary suppression of ventilation movements in some fish. It might thus act in part as a US which contributes, in conjunction with the electrical shock, to conditioning of the SR, but its occurrence does not affect the rate of acquisition of response to the moving LED-CS.

\section{Electrical (US)}

The US consisted of a $5 \mathrm{~mA}$ (rms), $0.5-\mathrm{sec}$ pulse of $60-\mathrm{Hz}$ constant current which was controlled by the microprocessor. The shock was delivered between steel electrodes which were located on either side of the posterior region of the fish's body (not shown in Fig. 1). The shock disrupted ventilation movements but the duration and magnitude of the response varied greatly. Each tank had a separate shock generator circuit [1].

\section{Preoperative conditioning}

Fish $(n=52)$ were administered a sequence of 6 sessions of conditioning trials over a period of 3 weeks. Sessions $1-5$ consisted of 10 trials each. The fish was placed in the tank in light for $5 \mathrm{~min}$ prior to the first trial in the session. Trials were initiated automatically by the microprocessor at random intervals of from 0.5 


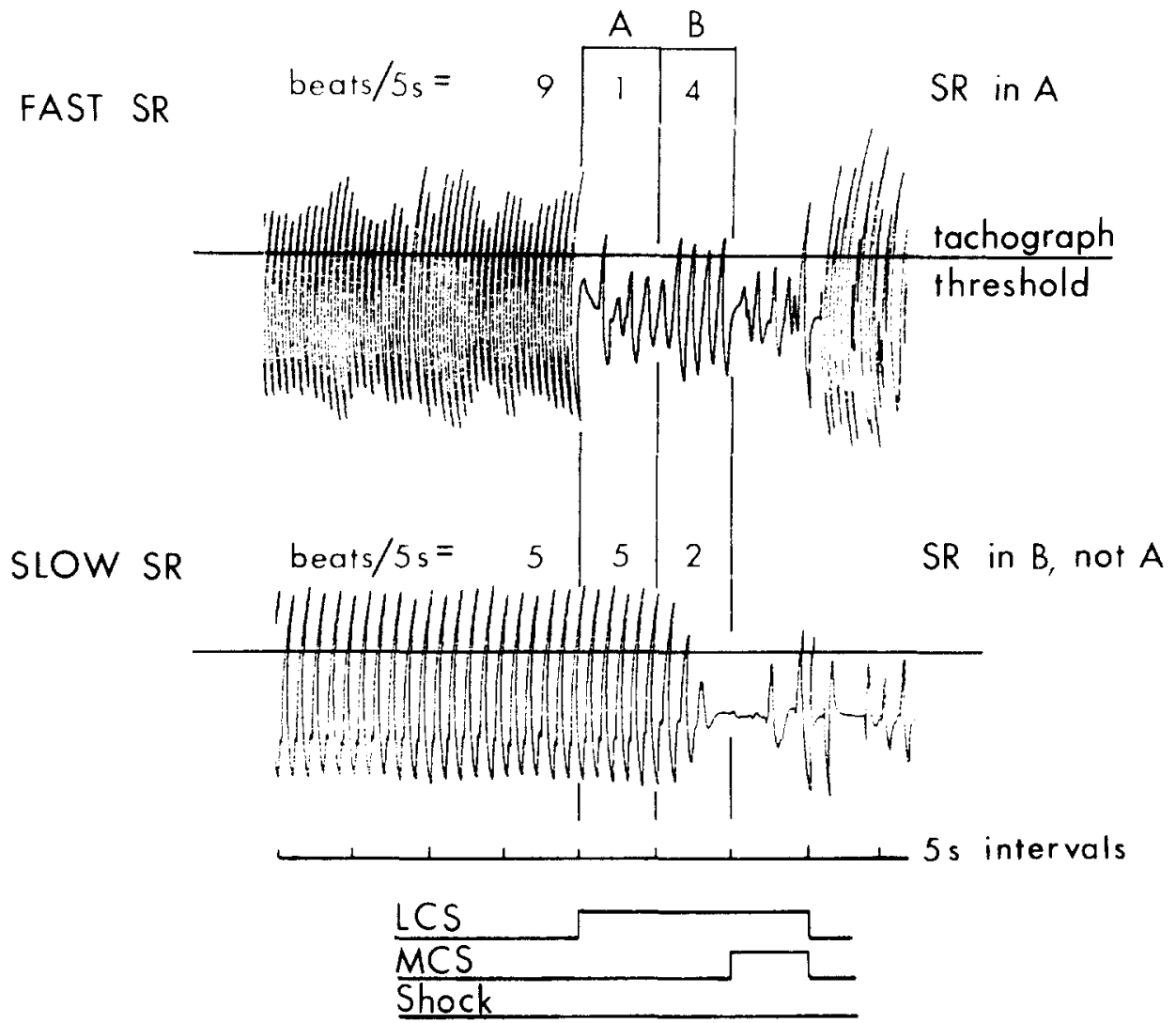

Fig. 3. Examples of fast and slow SRs. The illustrated fast SR represents an $89 \%$ deceleration (interval A) and the slow SR a 60\% deceleration (interval B) relative to the pretrial branchial rate. The tachograph threshold was drawn on the records but the tachograph pentrace was omitted for clarity. An example of the tachograph trace appears in Fig. 4.

to 3 min. The moving LED-CS was presented on the right $(R)$ or the left $(L)$ in pseudorandom order: RLLLRLRRRL.

Session 6 consisted of 6 conditioning trials (RLRLRL), which served as test trials, in which the SR was measured. Several blank trials were also administered to assess the occurrence of false-positive responses. In the blank trials, the microprocessor was activated but no trial stimuli were presented. Test and blank trials were initiated manually at times when the fish's branchial movements were regular. Trials in which the branchiogram was disrupted by body thrashing movements were not counted.

\section{Response criterion: fast and slow $S R$}

To facilitate analysis of the branchiogram, the brachial beats detected by the thermistor were registered on one channel of the recorder and counted with a tachograph, Grass model 7P4, on a separate channel. The gain of the thermistor 


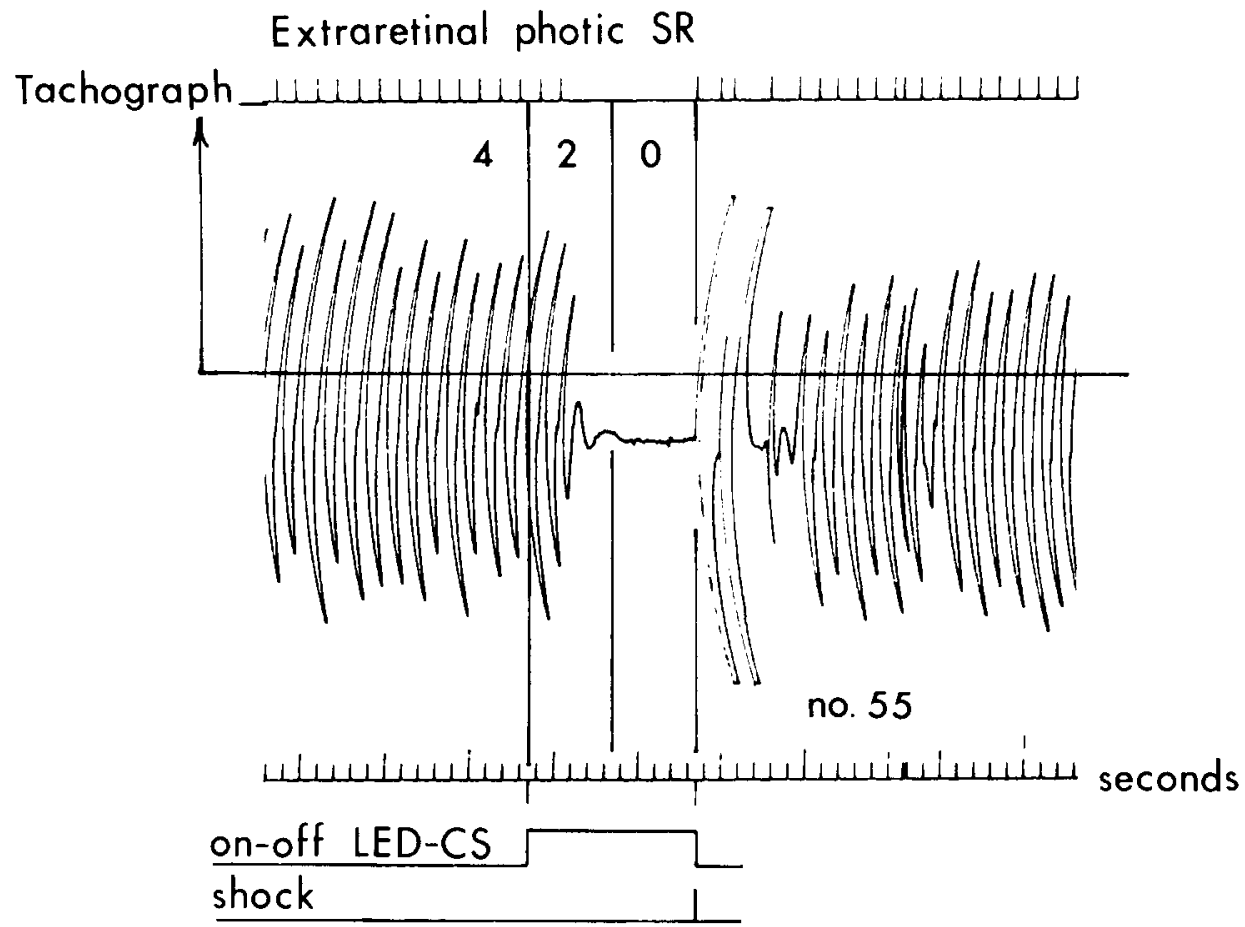

Fig. 4. An example of a fast SR to the on-off LED-CS by enucleate No. 55. It illustrates the minimum latency to response, approximately $3 \mathrm{sec}$, that was obtained in this highly responsive fish. The tachograph threshold was drawn on the record. An excursion of the branchial beat which exceeded the threshold resulted in a deflection of the tachograph pen.

recording was adjusted prior to a trial so that the tachograph triggered at approximately $50-75 \%$ of the amplitude of the beat from the chart centerline (Fig. 4).

To determine whether the fish made a SR and whether the SR was fast or slow, we counted the number of tachograph beats that occurred during the 5-sec interval preceeding the onset of the trial, and the numbers during the first $5 \mathrm{sec}$ (interval A) and the second $5 \mathrm{sec}$ (interval B) of the trial. The percent change in the rate in $A$ and in $B$, relative to the pretrial rate, was calculated. In the record shown at the top of Fig. 3, for example, the change in $A=100$ [ $(9$ minus 1) $/ 9]=89 \%$. A deceleration of greater than $30 \%$ in interval A or B was accepted as a SR, as false positives of that magnitude occurred in less than $3 \%$ of the blank trials. The SR in a test or blank trial was denoted as fast when the criterion was reached in interval A. If the criterion was reached in B but not in A, the SR was classified as slow.

\section{Experimental subjects and enucleation}

Thirty-two fish showing a mean deceleration in interval A of greater than $30 \%$ in the 6 test trials of the sixth preoperative session were randomly assigned 
to 4 groups $(\mathrm{n}=8 \mathrm{each})$, two of which received bilateral enucleation and two a sham operation [2]. In the sham surgery, the fish was anesthetized and the conjuctiva was slit but the tissues within the orbit were not otherwise disturbed.

\section{Postoperative conditioning}

The fish were administered a session of 14 conditioning trials in light or in darkness on postoperative Day 7, 10, 14, 17 and 21. The fish that were kept in darkness were illuminated only by the left and right LED rings. The first 10 trials in a session were initiated automatically. The final 4 trials were test trials initiated by the investigator in the sequence of RLRL to assess the occurrence of fast and slow SRs. A blank trial was administered prior to most test trials to assess the occurrence of false positives.

\section{Data analysis}

Occurrences of fast and slow SRs in the postoperative sessions were tabulated at the end of the experiment. The ink-trace record for each trial was coded and the identity of the fish and the session was revealed after the data was judged to be useable and the branchial beat-rates were measured. A few trials were found to have been inadvertently omitted and the records for others were eliminated owing to illegibility of the branchiogram. The number of SRs and the number of trials that were included in the analyses are presented as SR per opportunity ratios (SR/OPP).

The data for each session were reduced by calculating the mean probability of a SR, fast or slow, for each group. The individual's SR probability was obtained by dividing the number of SRs that it made in the session by the number of trials in which a SR could have occurred. The preoperative test trial and blank trial SR-probabilities of the 4 groups were contrasted with a two-way ANOVA. The primary analysis of postoperative data consisted of an ANOVA for two grouping factors, Vision (control vs enucleate) and Lighting (light vs darkness) and two within factors, Session (5) and Trial (test vs blank). BMDP statistical methods were used throughout the analyses [3].

\section{Results}

The groups had similar SR/OPP ratios in the preoperative test session (Table I), and the between-group differences in the SR-probability for test and blank trials were not significant (Table II). Enucleation virtually eliminated responding in test trials, and darkening the experimental tank impaired responding in control fish. These results are supported by the primary ANOVA which showed the mean SR-probability varied with the Vision factor, $F(1,28)=255.1$, $P<0.001$, and the Lighting factor, $F(1,28)=14.8, P<0.001$ ), and that Lighting interacted with Vision, $F(1,28)=19.2, P<0.001$. The type of trial was significant 


\section{TABLE I}

The SR/OPP for the preoperative session and the five postoperative sessions in Experiment 1

\begin{tabular}{|c|c|c|c|c|c|}
\hline \multirow[t]{2}{*}{ Trial } & \multirow[t]{2}{*}{$S R^{*}$} & \multicolumn{2}{|c|}{ Control groups } & \multicolumn{2}{|c|}{ Enucleated groups } \\
\hline & & Light & Dark & Light & Dark \\
\hline \multicolumn{6}{|c|}{ Preoperative } \\
\hline \multirow[t]{2}{*}{ Test } & $F$ & $36 / 48$ & $44 / 48$ & $39 / 48$ & $42 / 48$ \\
\hline & $\mathrm{S}$ & $0 / 12$ & $1 / 4$ & $1 / 9$ & $0 / 6$ \\
\hline \multirow[t]{2}{*}{ Blank } & $\mathrm{F}$ & $0 / 18$ & $1 / 18$ & $0 / 18$ & $0 / 18$ \\
\hline & $\mathrm{S}$ & $0 / 18$ & $0 / 17$ & $0 / 18$ & $1 / 18$ \\
\hline \multicolumn{6}{|c|}{ Postoperative } \\
\hline \multirow[t]{2}{*}{ Test } & $\mathrm{F}$ & $141 / 159$ & $70 / 159$ & $4 / 158$ & $2 / 154$ \\
\hline & $\mathrm{S}$ & $8 / 18$ & $21 / 89$ & $10 / 154$ & $5 / 152$ \\
\hline \multirow[t]{2}{*}{ Blank } & $\mathrm{F}$ & $4 / 113$ & $4 / 98$ & $3 / 120$ & $1 / 121$ \\
\hline & $\mathbf{S}$ & $2 / 109$ & $4 / 94$ & $6 / 117$ & $7 / 120$ \\
\hline
\end{tabular}

* Fast (F) or slow (S).

\section{TABLE II}

The mean probability of a fast or slow SR for test trials and blank trials (in parentheses) in the pre-and postoperative sessions of Experiment 1

\begin{tabular}{|c|c|c|c|c|}
\hline \multirow[t]{2}{*}{ Session } & \multicolumn{2}{|c|}{ Control groups } & \multicolumn{2}{|c|}{ Enucleated groups } \\
\hline & Light & Darkness & Light & Darkness \\
\hline Preoperative & $0.75(0.00)$ & $0.92(0.04)$ & $0.81(0.00)$ & $0.87(0.04)$ \\
\hline \multicolumn{5}{|l|}{ Postoperative } \\
\hline 1 & $0.84(0.08)$ & $0.28(0.00)$ & $0.03(0.04)$ & $0.03(0.00)$ \\
\hline 2 & $0.97(0.00)$ & $0.47(0.04)$ & $0.03(0.04)$ & $0.07(0.04)$ \\
\hline 3 & $1.00(0.00)$ & $0.65(0.10)$ & $0.09(0.12)$ & $0.00(0.08)$ \\
\hline 4 & $0.90(0.08)$ & $0.69(0.04)$ & $0.00(0.07)$ & $0.03(0.10)$ \\
\hline 5 & $0.99(0.08)$ & $0.72(0.12)$ & $0.06(0.04)$ & $0.03(0.04)$ \\
\hline
\end{tabular}

as a main effect, $F(1,28)=232.8, P<0.001$, and as a factor interacting with Vision, $F(1,28)=262.0, P<0.001$, Lighting, $F(1,28)=19.2, P<0.001$, and with Vision $\times$ Lighting, $F(1,28)=19.2, P<0.001$. Session was significant only as a main effect, $F(4,112)=4.17, P<0.004$.

An ANOVA on the Lighting factor for the enucleates showed no significant differences related to ambient light level, Trial-type or Session. For the controls 
a similar ANOVA indicated significant Lighting, $F(1,14)=19.9, P<0.001$, Session, $F(4,56)=3.97, P<0.007$, and Trial-type effects, $F(1,14)=282.9$, $P<0.001$, and a significant Trial $\times$ Lighting interaction, $F(1,14)=22.0$, $P<0.001$. The blank trial SR-probability did not differ significantly between the light- and dark-control groups. The change to conditioning in darkness then had no detectable effect on false positive SRs. The test trial SR-probability showed a marginal Lighting effect, $F(1,8)=5.7, P<0.06$. The 8 fish that were conditioned in darkness showed a wide variation in SR probability within and between sessions but most resumed responding by the third session.

These results indicated that the responding to the moving LED-CS depends upon a functional visual system and that the stimulus is ineffective in evoking the branchial SR extraretinally. No extraretinal responding could be demonstrated statistically, and subjective evaluation of the data gave no reason to question the results of analyses. The lack of positive SRs precluded analysis of whether the enucleates showed more slow than fast SRs. Conditioning in darkness impaired responding in a least some control fish, possibly by disrupting visual attention or recognition of the CS. Whether the procedure in some way inhibited responding in the enucleates cannot be determined from this experiment, but it seems unlikely that the change to darkness was responsible for the complete block of positive SRs.

\section{EXPERIMENT 2. RESPONSE TO AN ON-OFF LED-CS PRESENTED IN DARKNESS}

Enucleated goldfish respond to a CS consisting of a small spot of white or colored light that is turned on in darkness [2]. This observation in conjunction with the results of Experiment 1 suggested that an enucleate might detect the light from the LED ring but not its movement. To examine this possibility, a CS consisting of the light from one ring which was turned on and off but not moved was investigated. In addition, to obtain increased extraretinal photosensitivity, the fish were conditioned in darkness pre- and postoperatively.

\section{Method}

The upper LED ring was used to produce an on-off CS which was paired with the mechanical CS and the electrical US as described in Experiment 1. The ring was kept off during the intertrial interval and turned on and off in successive $250 \mathrm{msec}$ intervals during the $15 \mathrm{sec}$ trial (Fig. 2). The lower ring was never illuminated.

Forty fish were administered preoperative conditioning sessions as described in Experiment 1 but in darkened tanks. Eighteen fish selected following the 6-trial (RLRLRL) preoperative test session, were randomly assigned to two groups that received bilateral enucleation $(n=9)$ or the sham operation $(n=9)$. A post- 
operative conditioning session was administered in darkened tanks on Days 7,10 , $14,17,21$ and 24. The enucleates received 6 test trials per session (RLRLRL). For the controls the session was terminated when the individual was seen clearly to respond to right $(\mathrm{R})$ and left $(\mathrm{L})$ side presentations of the on-off CS or after a total of $3 \mathrm{R}$ and $3 \mathrm{~L}$ trials had been delivered. In addition several blank trials were recorded for each fish in every session. The data were tabulated and analyzed as described in Experiment 1.

Results

Preoperatively the two groups showed similar SR/OPP ratios (Table III) and mean SR-probabilities (Table IV). Following surgery the SR/OPP in test trials was greatly decreased for the enucleates but not the controls and the ratio in blank trials remained low for both groups (Table III). The SR-probability data for the two groups (Table IV) were contrasted using an ANOVA for one grouping factor, vision (control vs enucleate) and two within factors, Session (6) and Trial (test vs blank). The analysis showed significant Vision, $F(1,16)=203.9$, $P<0.001$, Trial, $F(1,16)=178.5, P<0.001$, and Trial $\times$ Vision effects, $F(1$, $16)=129.7, P<0.001$, but no significant session effect. An ANOVA on the trial factor for each group revealed a significant difference in SR-probability between test and blank trials for the control group, $F(1,8)=200.2, P<0.001$, but not for the enucleated group, $F(1,8)=4.1, P<0.08$.

The foregoing analyses suggest that responding to the on-off LED-CS was completely blocked following enucleation, but subjective evaluation of the data indicated that a few individuals selectively responded to the stimulus in some trials

TABLE III

The $S R / O P P$ for the preoperative session and the 6 postoperative sessions in Experiment 2

\begin{tabular}{lccc}
\hline Trial & $S R$ & Control group & Enucleated group \\
\hline $\begin{array}{l}\text { Preoperative } \\
\text { Test }\end{array}$ & F & & \\
& S & $48 / 54$ & $47 / 54$ \\
Blank & F & $2 / 6$ & $6 / 7$ \\
& S & $0 / 23$ & $0 / 24$ \\
Postoperative & & $0 / 23$ & $1 / 24$ \\
Test & F & & \\
& S & $128 / 171$ & $19 / 303$ \\
Blank & F & $12 / 43$ & $27 / 284$ \\
& S & $3 / 133$ & $9 / 241$ \\
& & $8 / 130$ & $10 / 232$ \\
\hline
\end{tabular}


TABLE IV

The mean probability of a fast or slow SR for test trials and blank trials (in parentheses) in the pre-and postoperative sessions of Experiment 2

\begin{tabular}{lll}
\hline Session & Control group & Enucleated group \\
\cline { 2 - 2 } & Test & Test \\
\hline Preoperative & $0.89(0.00)$ & $0.87(0.04)$ \\
Postoperative & & \\
1 & & \\
2 & $0.90(0.07)$ & $0.07(0.04)$ \\
3 & $0.93(0.03)$ & $0.13(0.07)$ \\
4 & $0.89(0.00)$ & $0.10(0.09)$ \\
5 & $0.89(0.07)$ & $0.06(0.04)$ \\
6 & $0.92(0.00)$ & $0.23(0.07)$ \\
& $0.82(0.07)$ & $0.23(0.10)$
\end{tabular}

and thus must have perceived it. The most striking examples occurred in the last two sessions in which the mean SR-probability was relatively high (Table IV). The strongest suspected responder, enucleate No. 55, showed slow SRs, one of which is illustrated in Fig. 3, and fast SRs which had a latency of longer than $3 \mathrm{sec}$. Subsequent study supported the impression that the on-off LED-CS is an extraretinal photic stimulus.

\section{Post-hoc manipulations}

Following the main experiment the fish were administered additional conditioning over a period of several weeks to see if positive SRs in the enucleates could be increased. The procedure was simplified by presenting the on-off LED-CS only on the fish's right side, the duration of the CS was shortened to $10 \mathrm{sec}$, and the MCS was omitted. Several of the enucleates showed signs of responding to the $\mathrm{CS}$ but strong consistent responding comparable to that of eyed goldfish was obtained in only one, No. 55. The SR latency varied from trial to trial but it was never less than several seconds in duration (Fig. 4).

Fish No. 55 also readily responded to a 10 -sec CS consisting of a white light (W-CS). The W-CS, which resembled the CS used in Davis and Benloucif [2], was produced by an incandescent lamp (G.E. 1815;24 VDC) which was mounted next to the LED display on the fish's right side. The W-CS was approximately 200 times brighter ( $250 \mathrm{ft}$.-lamberts) than the on-off LED-CS (1.3 ft.-lamberts), measured using the reflected-light method (Experiment 1). The fish consistently responded to either $\mathrm{CS}$, presented in varying order within a session, but it never responded in trials with the moving LED-CS (Fig. 5). 

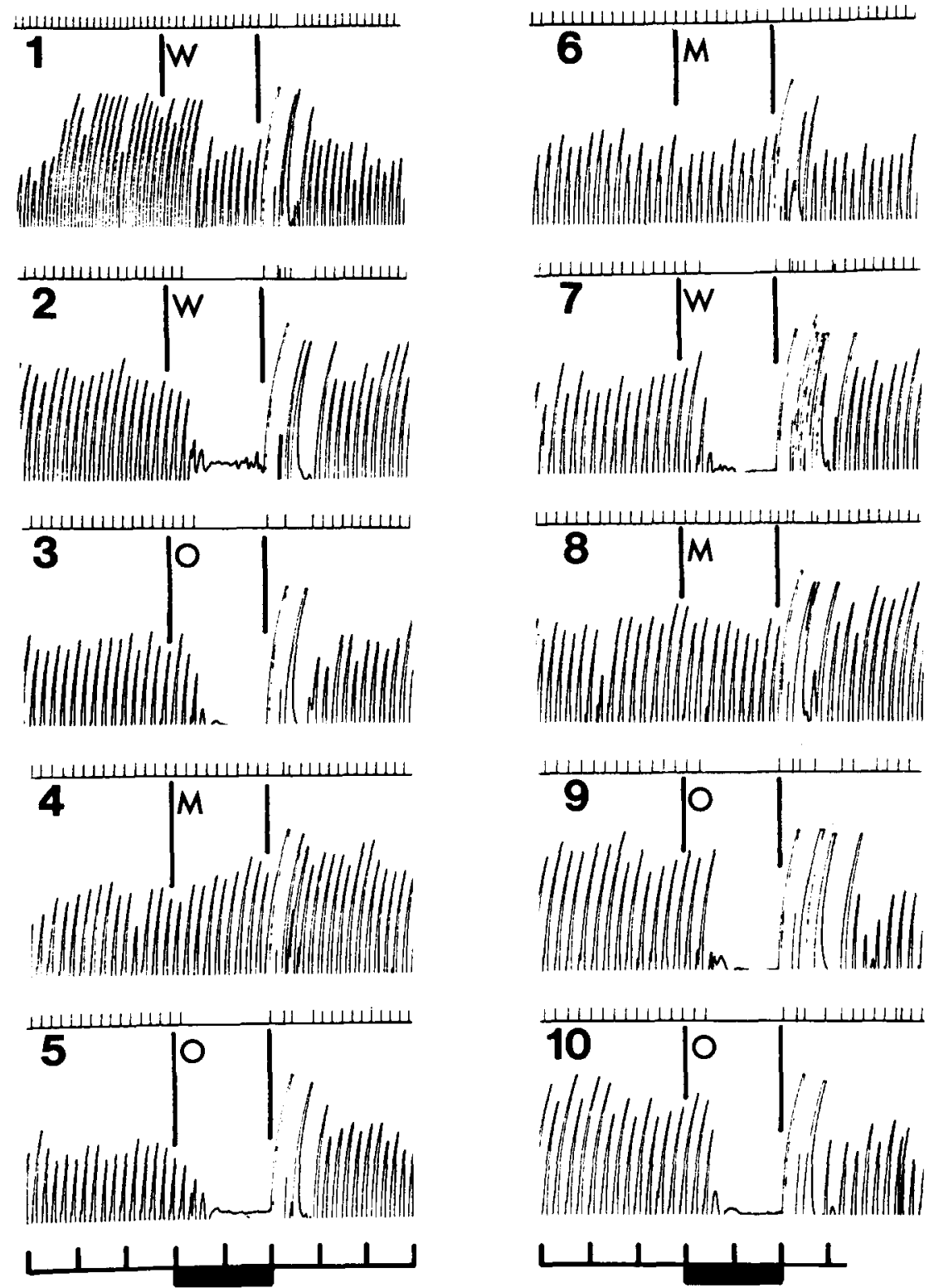

Fig. 5. Performance of enucleate No. 55 in a session of 10 trials with various types of CS. Following the initial trial the fish responded to the W-CS (W) and the on-off LED-CS $(O)$ but not the moving LED-CS (M). The electric shock US was delivered at the end of the $10 \mathrm{sec}$ CS interval (solid bar). The record for each trial consists of the portion of the branchiogram above the chart centerline and the tachograph trace.

Responding to the on-off LED and the W-CS was blocked in trials in which an opaque mask was placed in the light path. Turning on the overhead adapting light also blocked the on-off LED response but responding to the much brighter W-CS, although greatly decreased, was not eliminated. Varying the adapting light, 
in different sessions, by controlling the voltage, revealed that less than $2 \mathrm{ft}$.-lambert (40 volts) of overhead light was sufficient to block the on-off LED response.

\section{DISCUSSION}

The foregoing results confirm that extraretinal photic input can be conditioned to electric shock in at least some bilaterally enucleated goldfish and that such extra-retinal sensitivity is then a potential source of error in optic nerve regeneration experiments. A light stimulus that evokes a branchial SR in enucleates might do so in fish in which one eye is removed and the other optic nerve is crushed, as by Davis and Benloucif [2], and the occurrence of extraretinal photoresponses could result in overestimating the rate of regeneration of the damaged nerve. We have examined approximately 50 additional enucleates using the relatively dim, on-off LED-CS and a bright, W-CS. Although most showed no sign of responding, consistent responding similar to that of No. 55 in Experiment 2 was obtained in only two fish. None of these otherwise highly responsive fish was seen to respond to the moving LED-CS. Although the experimental and biological variables that control conditioning of extraretinal photic are poorly understood, the available data indicate that extraretinal photoresponding can be circumvented by using the moving LED-CS.

The evidence in Experiment 2 that the enucleated goldfish can detect the light from the LED ring in an otherwise darkened environment is consistent with reports that the threshold of behavioral sensitivity to extraretinal photostimulation in fishes is very low $[6,8]$. The enucleate can be inferred to perceive the light only very diffusely, since the flickering up-down movement of the ring evoked no response. The enucleate might respond to a CS consisting of a more extensive shift in the LED ring as, for example, from below to above the head, which could result in stimulating different extraretinal photoreceptors. As very dim overhead illumination blocked responding to the on-off LED-CS in enucleate No. 55, the addition of an adapting light might suffice to block extraretinal photoresponding to a change-of-intensity CS.

Conditioning of extraretinal photostimulation has not been extensively investigated. Such stimulation has been reported to evoke behavioral responses consisting of changes in swimming activity in various fishes [5, 6, 10]. Early experiments in the cyprinid, Phoxinus suggest that enucleates can learn to approach the source of bright overhead illumination for food [9]. More recently, Fenwick [4] found that enucleation blocks acquisition of conditioning of a light CS to shock in an active shock avoidance task. Whether enucleation impairs retention of light-shock conditioning was not reported. Fenwick postulated that the neuronal circuitry that mediates extraretinal photic input is such that an extraretinal photic CS cannot activate instrumental reponses. Our findings that the circuitry is clearly susceptible to classical conditioning do not necessarily 
contradict Fenwick's proposal. It is possible that active shock avoidance is a less sensitive indicator of light detection than food reinforced approach [9] or classical conditioning of branchial suppression. Although many individual goldfish would probably have to be screened to obtain a sufficient number of experimental subjects, classical conditioning could be a useful method for psychophysical investigations of extraretinal photoreception in fishes as well as in optic nerve regeneration studies.

\section{ACKNOWLEDGEMENT}

This work was supported by United States Environmental Protection Agency, Grant 808064.

\section{REFERENCES}

1 Davis, R.E., A method of restraining goldfish during conditioning of electric-shock-induced suppression of breathing movements. Behav. Res. Meth. Instr., 13 (1981) 650-652.

2 Davis, R.E. and Benloucif, S., Behavioral investigation of neurotoxicity: the effects of colchicine, lumicolchicine and vincristine sulfate on goldfish optic nerve regeneration, Neurotoxicology, 2 (1981) 419-430.

3 Dixon, W.J., Brown, M.B., Engelman, L., Frane, J.W., Hill, M.A., Jennrich, R.I. and Toporek, J.D., BMDP Statistical Software, Univ. California Press, Berkeley, CA, 1981, pp. 347-349, 368-372.

4 Fenwick, J.C., Effects of pinealectomy and bilateral enucleation on the phototactic response and on the conditioned response to light of the goldfish, Carassius auratus L., Canad. J. Zool., 48 (1970) 175-182.

5 Jones, F.R.H., The behavior of minnows in relation to light intensity, J. exp. Biol., 33 (1956) 271-281.

6 Kavaliers, M., Retinal and extraretinal entrainment action spectra for the activity rhythms of the lake chub, Couesius plumbeus, Behav. Neural. Biol., 30 (1980) 56-67.

7 Northmore, D.P.M. and Yager, D., Psychophysical methods for investigations of vision in fishes. In M.A. Ali (Ed.), Vision in Fishes, Plenum Press, New York, 1975, pp. 689-704.

8 Underwood, H., Extraretinal photoreception. In E.H. Burtt (Ed.), The Behavioral Significance of Color, 1979, Garland STPM Press, NY, pp. 129-178.

9 Scharrer, E., Die lichtempfindlichkeit blinden Eleritzen, Z. Vergl. Physiol. 7 (1928) 1-38.

10 van Veen, T.H., Hartwig, H.G. and Müller, K., Light-dependent motor activity and photonegative behaviour in the eel (Anguilla anguilla L.), J. comp. Physiol., 111 (1976) 209-219. 